Laboratory Investigation (2006) 86, 979-980.

doi:10.1038/labinvest.3700467

\section{Mind the gap}

Like all epithelia, the cells that line the gastrointestinal tract turn over frequently. In the case of the mammalian small intestine, the epithelia proliferate in the crypts and differentiate from secretory to absorptive cell as they migrate into the villus. At the end of this process, the epithelia are shed from the villus and replaced by a new wave of cells. While it is clear that this shedding and replacement process occurs continuously, some estimate that more than one million cells are shed each day, and the essential transport and barrier functions of the intestinal epithelium are not compromised. This is critical, as loss of barrier function is associated with intestinal disease. ${ }^{1}$ Thus, the question of how individual epithelial cells are shed while an intact epithelial barrier is maintained is a mystery of physiological, and perhaps pathophysiological, significance.

Several groups have previously studied the process of epithelial cell shedding by light and electron microscopy using animal models. ${ }^{2,3}$ These studies show that not even transient leaks are formed during the cell shedding process. One electron microscopy-based study made it appear that shedding cells are pushed out of the epithelium as the adjacent cells form new junctions beneath the extruding cell. ${ }^{2}$ In contrast, a more recent study using time-lapse light microscopy of living mouse mucosa made the surprising observation that a gap is formed as a cell shed. The study showed that barrier function is maintained at the sites of these cell-free gaps, despite the observation that $\sim 3 \%$ of cell 'positions' were gaps. ${ }^{3}$ What may be most remarkable about these observations is that, despite of the staggering number of small intestinal biopsies collected and examined in hospitals throughout the world, no such gaps have been reported in human intestine. How can this be? Are the observations made in rodents simply not applicable to humans? Could such profound species differences exist?

In this issue of Laboratory Investigation, Bullen et $\boldsymbol{a l}{ }^{4}$ (p. 1052) considered the hypothesis that the reason for the absence of apparent gaps in routinely processed tissues is that processing techniques make it difficult to identify gaps. They therefore began with an analysis of alternative processing approaches. Using an approach designed to minimize handling of the epithelial surface, Bullen et al were able to identify gaps in formalin-fixed, paraffin-embedded human tissues that were indistinguishable from those seen in mouse. This new tissue preparation technique allowed Bullen et al to characterize a number of morphological features of shedding cells. For example, based on recent report of purse-string contractile actin rings effecting closure of small oligocellular wounds, ${ }^{5}$ Bullen et al asked if actomyosin contraction might contribute to closure of the gaps left after cell shedding. The data suggest that this is the case. Bullen et al also asked if shedding triggers apoptosis, that is, anoikis, or, conversely, if apoptosis triggers shedding. While the data are not yet unequivocal, they favor the anoikis.

In addition to the exciting new data presented, this study raises a number of important questions regarding the mechanism and timing of events involved in cell shedding and renewal, and also provide new possibilities for identification of the acellular eosinophilic material that fills the gaps left by shed cells. Finally, and perhaps most intriguingly, the novel tissue processing technique developed may allow future detection of diseases in which defective cell shedding and renewal contribute to pathogenesis.

David Ronan Raleigh The University of Chicago

\section{References}

1 Clayburgh DR, Shen L, Turner JR. A porous defense: the leaky epithelial barrier in intestinal disease. Lab Invest 2004;84:282-291.

2 Madara JL. Maintenance of the macromolecular barrier at cell extrusion sites in intestinal epithelium: physiological rearrangement of tight junctions. J Membr Biol 1990;116:177184.

3 Watson AJ, Chu S, Sieck L, et al. Epithelial barrier function in vivo is sustained despite gaps in epithelial layers. Gastroenterology 2005;129: 902-912.

4 Bullen T, Forrest S, Campbell F, et al. Characterization of epithelial cell shedding from the luminal surface of the human small intestine; a process involving phosphorylation of myosin light chains. Lab Invest 2006;86:10521063.

5 Russo JM, Florian P, Shen L, et al. Distinct temporal-spatial roles for rho kinase and myosin light chain kinase in epithelial purse-string wound closure. Gastroenterology 2005;128:9871001. 
Endogenous pancreatitis from inactivation of the serum response factor gene

Over the past 2 years, the pages of this journal have given significant attention to understanding development of the pancreas, and to experimental models of pancreatitis. In the first instance, $P d x 1$ (Pancreatic and duodenal homeobox 1) has been explored as a key gene regulating differentiation of progenitor cells into both the exocrine and endocrine lineages of the pancreas. ${ }^{1}$ In the second instance, chemical models of pancreatitis usually have to be employed. Linkage of the two processes of ontogenesis and pancreatitis has not been made. In this issue, Miralles et $\boldsymbol{a l}^{2}$ (p. 1020) demonstrate that a transcription factor that acts at the confluence between signalling pathways involved in cellular differentiation, growth, and death may have a key role in the pancreas-not in pancreatic development as hypothesized, but in prevention of pancreatitis. This factor is serum response factor (SRF), which is a member of the MADS box family that binds the CC(A/T)6GG sequence known as the CArG box. In general, mice rendered null for SRF are embryonic lethal, owing to failed development of the mesodermal layer; overexpression of a dominant negative form of SRF also damages embryogenesis. In specific example, targeted deletion of SRF in the heart leads to embryonic lethality; overexpression of functional SRF in the heart leads to cardiac hypertrophy. As SRF has been identified as a transcriptional regulator of the expression of antiapopotic genes, it can be posited that SRF plays a key role in programmed cell death.

Several genes containing the CArG box in their transcriptional regulatory region are expressed in the pancreas, and SRF is produced in $\beta$-cells in response to a glucose challenge. Hence, the question can be asked about the role of SRF in pancreatic development and maintenance. Accordingly, Miralles et al first demonstrate that SRF is indeed expressed in the murine pancreas throughout pancreatic development and in the adult pancreas, with higher levels of SRF expression in islet cells. A conditional inactivation of the SRF gene in the pancreas was then achieved by crossing mutant mice carrying a floxed Srf allele with $p P d \times 1$-Cre transgenic mice. The $p P d x 1$-Cre mice expressed the recombinase Cre under the control of the promoter of the $P d x 1$ gene, hence enabling generation of mutant mice with specific inactivation of the Srf gene in the entire pancreas. The authors confirmed that excision of the SRF gene was complete in the pancreas. Interestingly, the pancreas developed normally, so that at birth and during the neonatal period there was no evident pancreatic structural or functional defect induced by SRF absence. However, a severe pancreatitis ensued at the time of weaning, complete with interstitial edema, inflammation, and fibrosis, with elevation of serum levels of amylase and lipase. This led to total destruction of the exocrine pancreas, with its eventual replacement at 4 months of age by adipose tissue. The structure and function of the endocrine islets was not adversely affected. In exploring potential mechanisms for the pancreatitis, the authors found only minimal increased expression of proapoptotic genes in the Srf-inactivated pancreata. Rather, substantially increased expression of the proinflammatory transcriptional mediator NF- $\kappa \mathrm{B}$ was identified, implicating a necroinflammatory rather than apoptotic cause of the exocrine pancreatitis. This last finding also is of interest given that enhanced expression of NF- $\kappa \mathrm{B}$ may paradoxically protect islets from apoptotic destruction. ${ }^{3}$

At the very least, this report provides a potentially new model of endogenous exocrine pancreatitis, with opportunity to explore the pathogenesis of tissue destruction from the outset of the inflammatory process without a requirement for exposure to chemical toxins. More fundamental is the potential to explore the relationship between SRF and the regulation not only of cell growth and death but also the inflammation that may simultaneously occur. This murine model may thus have broader impact than simply exploring the mechanisms underlying pancreatitis.

\section{References}

1 Tang DQ, Lu S, Sun YP, et al. Re-programming liver stem-WB cells into functional insulinproducing cells by persistent expression of Pdx1 and Pdx1-VP16 mediated by lentiviral vectors. Lab Invest 2006;86:83-93.

2 Miralles F, Hebrard S, Lamotte L, et al. Conditional inactivation of the murine serum response factor in the pancreas leads to severe pancreatitis. Lab Invest 2006;86:1020-1036.

3 Hasel C, Bhanot UK, Heydrich R, et al. Parenchymal regression in chronic pancreatitis spares islets reprogrammed for the expression of NFkappaB and IAPs. Lab Invest 2005;85:1263-1275. 